Int. J. Dev. Biol. 60: 175-179 (2016)

doi: $10.1387 / \mathrm{ijdb} .160045 \mathrm{mo}$

\title{
Platelet derived growth factor B gene expression in the Xenopus laevis developing central nervous system
}

\author{
KETY GIANNETTI ${ }^{1}$, DEBORA CORSINOVI ${ }^{1}$, CRISTINA ROSSINO ${ }^{1}$, IRENE APPOLLONI ${ }^{2,3}$, PAOLO MALATESTA ${ }^{2,3}$ \\ and MICHELA ORI*,1 \\ ${ }^{1}$ Dipartimento di Biologia, Università di Pisa, ${ }^{2} I R C C S-A O U$ San Martino-IST, Genoa and \\ ${ }^{3}$ Department of Experimental Medicine (DiMES), University of Genoa, Italy
}

\begin{abstract}
Platelet-derived growth factor B (PDGF-B) belongs to the mitogen and growth factor family and like the other members it has many roles in cell differentiation, proliferation and migration during development, adult life and in pathological conditions. Among them it has been observed that aberrant PDGF signalling is frequently linked to glioma development and progression, and $\boldsymbol{P d g f - b}$ over-expression in mouse neural progenitors leads to the formation of gliomas. Despite this evidence, the mechanisms underlying PDGF-B driven tumorigenesis and its role during brain development are not fully understood. In order to contribute to clarifying possible new roles of pdgf-b signalling, we present here the embryonic gene expression pattern of pdgf-b, so far unknown in early vertebrate development. By using Xenopus laevis as a model system we performed qRT-PCR and whole mount in situ hybridization. Pdgf-b mRNA is expressed in discrete regions of the developing central nervous system, in the cranial nerve placodes and in the notochord. We also compared the gene expression of $p d g f-b$ with that of its receptor $p d g f r-\alpha$ suggesting so far unsuspected roles for this signalling pathway during the development of specific embryonic structures.
\end{abstract}

KEY WORDS: PDGF-B, PDGF receptor $\alpha$, central nervous system, neural crest, Xenopus laevis

Platelet-derived growth factor (PDGF) family comprises two tyrosine kinase receptors (PDGFR- $\alpha$ and $-\beta$ ) and four ligands (PDGF-A, $-B,-C$, and $-D)$ that form homodimers or the heterodimer $A B$ (Demoulin and Essaghir, 2014). The active ligand-receptor complex consists of two receptor chains associated with one dimeric ligand. While PDGFR- $\alpha$ binds to all PDGF isoforms except for PDGF-DD, PDGFR- $\beta$ binds only to PDGF-BB and -DD (Fig.1). Also a heterodimeric $\alpha \beta$ receptor has been reported that binds to PDGF-AB, -BB and possibly-CC and-DD (Demoulin and Essaghir, 2014). The members of this family have been extensively studied for more than 30 years in development, adult homeostasis and disease (Heldin, 2013, Heldin, 2014, Hoch and Soriano, 2003). Pdgf-a expression was recently characterized in mouse tissues and in early embryonic development, showing its involvement in developmental processes including gastrulation and craniofacial development (Andrae et al., 2014, Eberhart et al., 2008). On the contrary, for $p d g f-b$ the only information available mainly concern its role during embryonic angiogenesis (Hoch and Soriano, 2003, Leveen et al., 1994), and a complete gene expression pattern in vertebrate embryogenesis is still lacking. Functional studies using a knock out mouse for pdgf-b showed abnormal kidney glomeruli, heart and blood vessel dilation, anemia, thrombocytopenia, haemorrhages and perinatal death (Betsholtz, 1995, Leveen et al., 1994). However, in this mutant line, BB homodimer and $A B$ heterodimer were simultaneously abrogated, making difficult to precisely define the specific role of pdgf-b alone during embryogenesis. Furthermore, due to extensive perinatal haemorrhages, other possible phenotypes caused by the lack of pdgf-b in specific tissues could not be deeply investigated.

It is known that pdgf-b possesses a C-terminal basic retention motif, which binds to extracellular matrix components. The presence of this motif does not prevent binding to the receptor but limits the pdgf-b diffusion. When the retention motif is removed by intracellular or extracellular proteases, a soluble and diffusible pdgf-b form is released. Recently, the study of mutant mice harboring a pdgf- $b$ gene that lacks the retention motif shed light on its importance, showing that the mutant mice present an intermediary phenotype between wild-type and pdgf-b knock-out animals (Demoulin and

Abbreviations used in this paper: PDGF, platelet derived growth factor; qRT-PCR, quantitative reverse transcription-polymerase chain reaction; WISH, whole mount in situ hybridization; Xenopus, Xenopus laevis.

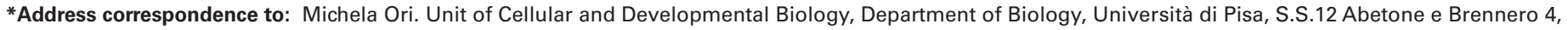
56127 Pisa, Italy. Tel: +39 050 2211484. Fax: +39050 2211495. E-Mail: michela.ori@ unipi.it

Accepted: 26 May 2016

ISSN: Online 1696-3547, Print 0214-6282 
Essaghir, 2014). The interplay between pdgf-bb and the extracellular matrix has been also demonstrated in many cell types, for instance, in dermal fibroblasts pdgf-b stimulates the synthesis of hyaluronan, which in turn is required for the mitogenic activity of pdgf-b, via the activation of CD44 receptor (Li et al., 2007).

Another interesting aspect of studying PDGF family function relies on the fact that over activity of PDGF signalling is associated with several malignant and non-malignant diseases (Heldin, 2013, Heldin, 2014). Among malignant ones it was shown that in gliomas, a group of heterogeneous and aggressive brain tumours, alteration of PDGF signalling is commonly observed (Calzolari and Malatesta, 2010); interestingly PDGF-Bover-expression alone was shown inducing oligodendroglioma, a specific subclass of glioma, from mouse neural progenitors, suggesting a crucial role for this molecule in tumorigenesis (Appolloni et al., 2009). Moreover recent data about the cellular mechanisms of PDGF-B driven glioma progression and maintenance indicate that PDGF may be required to override cell contact inhibition and promote glioma cell infiltration (Calzolari and Malatesta, 2010). Nevertheless not much is known about the molecular mechanisms activated downstream PDGF-B signalling. A deeper comprehension of pdgf-b role during development might help in shedding light on its mechanisms of action in modulating different aspects of cell behaviour also involved in tumorigenesis and tumour progression.

\section{Results and Discussion}

\section{PDGF-b is expressed during Xenopus laevis development}

The aim of this work was to describe the gene expression pattern of $p d g f-b$ during embryogenesis using Xenopus laevis as a model. Xenopus is a tetrapod from the same evolutionary branch

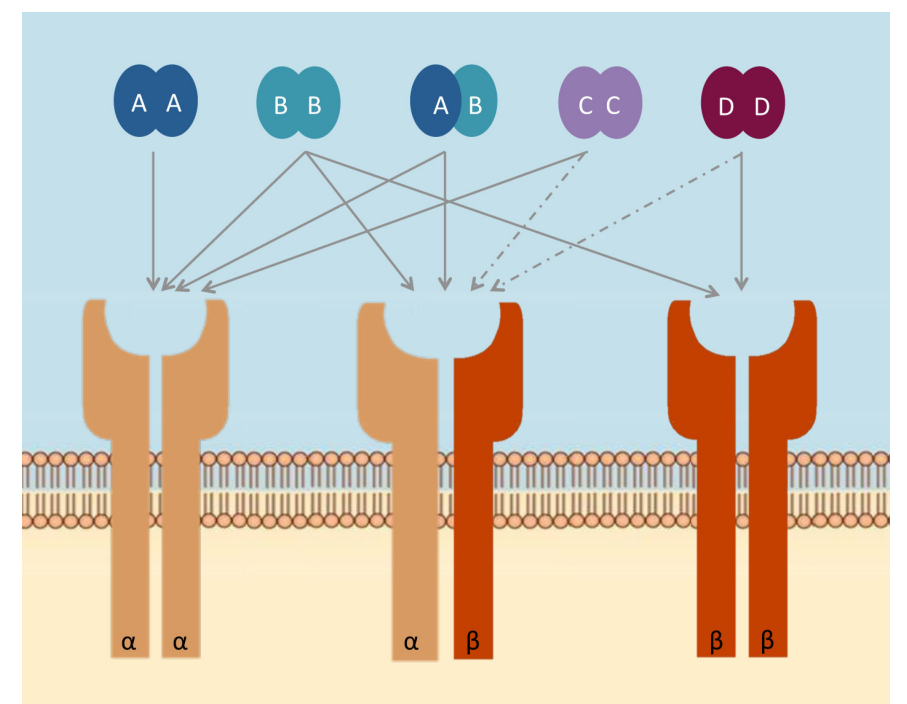

Fig. 1. Platelet derived growth factor (PDGF) family members and their interactions. PDGF family is composed by the four ligands $A, B, C$ and $D$ that need to dimerize in order to be active and bind to the receptor. They can homodimerize and the only known heterodimer is $A B$. The two receptors, $\alpha$ and $\beta$, are tyrosine kinases and have different affinity for the ligands. When the ligand-receptor complex forms, the intracellular domains of the receptors trans-phosphorylate each other, triggering the signal. Arrows indicate interaction, dashed arrows indicate hypothetical interactions.
40

35

30

25

20

15

10

10

5

st3

Fig. 2.The temporal expression pattern of $\boldsymbol{p d g} \mathbf{f b}$ revealed by qRT-PCR. Expression levels (y axis) of pdgf-b (red) and its receptor pdgfr- $\alpha$ (blue) at different developmental stages ( $x$ axis) were analyzed by qRT-PCR. The data are an average of three independent experiments, the values were normalized to odc and presented as mean \pm s.d. Expression of pdgf-b $m R N A$ reaches its maximum level as maternal transcript (stage 3), then drastically decreases during gastrulation (stage 10,5). A slight increase is observed during early neurulation (stage 13), however the expression becomes more robust at late neurula stage (stage 20) to decrease again in tailbud stages. Pdgfr- $\alpha$, is barely detectable at stage 3 and then rise firmly already during gastrulation, reaching its maximum level at stage 20.

of mammals and because of its external embryonic development, it represents a suitable model to study in details the expression pattern of a specific gene from the very early steps of embryogenesis. We firstly performed qRT-PCR comparing the relative expression level of $p d g f-b$ with that of its receptor $p d g f r-\alpha$ already described (Bae et al., 2014) (Fig. 2). Pdgf-b mRNA is present as a maternal transcript within the blastula at 4 cells stage (stage 3 according to Nieuwkoop and Faber, 1967). After the start of the zygotic transcription (stage 8), pdgf-b mRNA was barely detectable and it showed a low expression level both during gastrulation (stage 10.5) and neurulation (stage 13); on the contrary, pdgfr- $\alpha$ was abundantly expressed at these stages. During gastrulation in Xenopus, a crucial role for pdgf-a and pdgfr- $\alpha$ has been demonstrated (Damm and Winklbauer, 2011). As pdgf-bmRNA is extremely poorly expressed during gastrulation, this data corroborates the idea that in the early phases of development, the main role of PDGF signalling is played by the homodimer pdgf-aa via pdgfr- $\alpha$. This is in line with the observation that in pdgf-a morphants the prechordal mesoderm fails to spread during gastrulation and no compensatory mechanisms occurs (Damm and Winklbauer, 2011). At the end of neurulation (Stage 20), pdgf-bmRNAexpression level rose and then decreased again at late tailbud stages (stage 35) (Fig. 2).

In order to define the localization of $p d g f-b m R N A$, we performed whole mount in situ hybridization (WISH) using a digoxigenin labelled $p d g f-b$ antisense RNA probe on wild type Xenopus embryos at different stages of development (Figs. 3-4). We confirmed the presence of $p d g f-b$ as a maternal transcript at stage 3 , when the mRNA is localized in the animal pole (Fig. $3 A$ ). In the gastrulating embryo, pdgf- $b$ was faintly expressed in the animal pole in the deep layer of the ectodermal blastocoel roof (Fig. 3 B-B'). In these early phases of development the riboprobes could sometimes generate false positive signals due to the unspecific labelling of the blastocoel cavity. We therefore confirmed the specificity of $p d g f-b$ mRNA localization performing the same WISH experiment using a $p d g f-b$ sense riboprobe (Fig. $\left.3 A^{*}-B^{*}-B^{\prime *}\right)$. At the end of neurulation (stage 20-21) the gene expression pattern of $p d g f-b$ resulted 
more robust and localized (Fig. $3 \mathrm{C}$ ). At this stage, two stripes in the midbrain-hindbrain region of the central nervous system (CNS) are clearly defined and the placode of the facial nerve is labelled. We also observed a strongly labelled domain in anterior region of the neuroectoderm, possibly corresponding to the future telencephalon (Fig. $3 \mathrm{C}-\mathrm{H}$ ). At the same stage, pdgf- $b$ mRNA is also localized in the notochord (Fig. $3 \mathrm{C}$ inset). We then performed double WISH to better define pdgf-b expression territories in the CNS by using specific molecular markers to label respectively the midbrain-hindbrain boundary (engrailed2) and rhombomeres 3 and 5 (krox20). Our data showed that $p d g f-b$ mRNA localized in rhombomere 1 and rhombomere 3 of the hindbrain (Fig. 3 D-E). In order to define the identity of the most anterior $p d g f-b$ expression domain, we also performed double WISH with the retinal marker

Fig. 3 (right). Pdgf-b spatial expression pattern during early stages of development in Xenopus laevis embryos. (A) Pdgf-b maternal transcript is distributed in the animal pole (arrow) at four cell stage (stage 3), lateral view. (B) Lateral view of a stage 10,5 embryo showing the pdgf-b mRNA localization in the animal pole of the embryo (arrow). (B') Bisected gastrula showing the pdgf-b expression confined within the deep layer of the ectodermal blastocoel roof (arrowheads). (A*) WISH performed using a pdgf-b sense riboprobe on a stage 3 embryo, lateral view. (B*) Stage 10,5 lateral view WISH using the pdgf-b sense riboprobe: no signal is detected in the animal pole. (B'*) Bisected gastrula showing no unspecific labelling of the blastocoel cavity using the pdgf-b sense riboprobe. (C) Late neurula, (stage 20) embryo frontal view. Pdgf-b mRNA is localized in the CNS (midbrainhindbrain region: black and red arrowheads: the most ventral anterior region of neural tube: yellow arrow), the placode of the facial nerve (black arrow) and the notochord (inset). (D) Stage 20 frontal view double WISH: pdgf-b signal revealed in purple (black and red arrowheads), engrailed2 labelling the midbrain-hindbrain boundary and rombomere 1, in light blue. The two signals overlaps at the level of rhombomere 1. (E) Stage 20 frontal view double WISH: pdgf-b signal in purple (black and red arrowheads), krox20
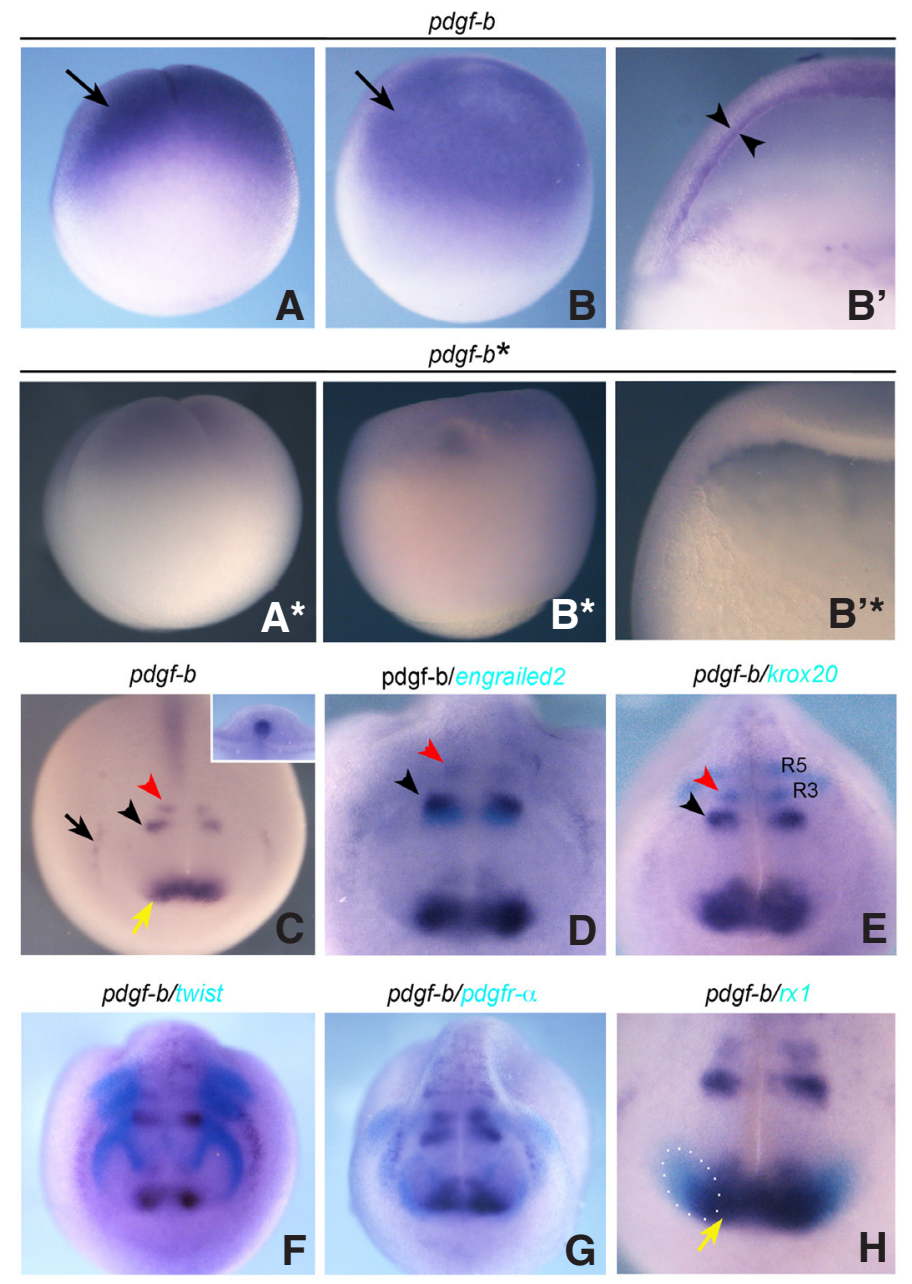

labelling rhombomeres 3 (R3) and 5 (R5), in light blue. The two signals overlaps faintly at the level of rhombomere 3. (F) Double WISH using twist, a NCC marker in light blue. NCC are in close proximity of the pdgf-b expression domain but never overlap it. (G) Double WISH using pdgfr- $\alpha$ in light blue. Pdgfr- $\alpha$ is expressed in migrating NCC. (H) Frontal view of an embryo processed with double WISH using rx1, a marker for the eyefield (dashed oval), revealing a partial overlap of the pdgf-b signal (yellow arrow) with an anterior and ventral domain of the eyefield.

Fig. 4. Pdgf-bexpression spatial distribution in Xenopus laevis embryo at tailbud stage. (A) Pdgf-b mRNA is present in the hindbrain (R1 and R3), the most ventral anterior region of the developing CNS (yellow arrow), the placode of the facial nerve (black arrow) and the notochord (inset, green arrowhead). (B-E) Double WISH was performed using different markers (the color code is described in Fig.3) at tailbud stage (stage 25). (B) Lateral view of an embryo processed by double WISH using twist as NCC marker. (C) Pdgfr- $\alpha$ is expressed by NCC, in close proximity with the pdgf-b mRNA expression domain. (D-D') Frontal and lateral views of the same embryo labelled to visualize rx1, a retinal marker (dashed oval identifies the eyefield) and pdgf-b. (E) A sagittal view of a half embryo bisected along the antero-posterior axis shows the expression of pdgf-b in the ventral telencephalon.
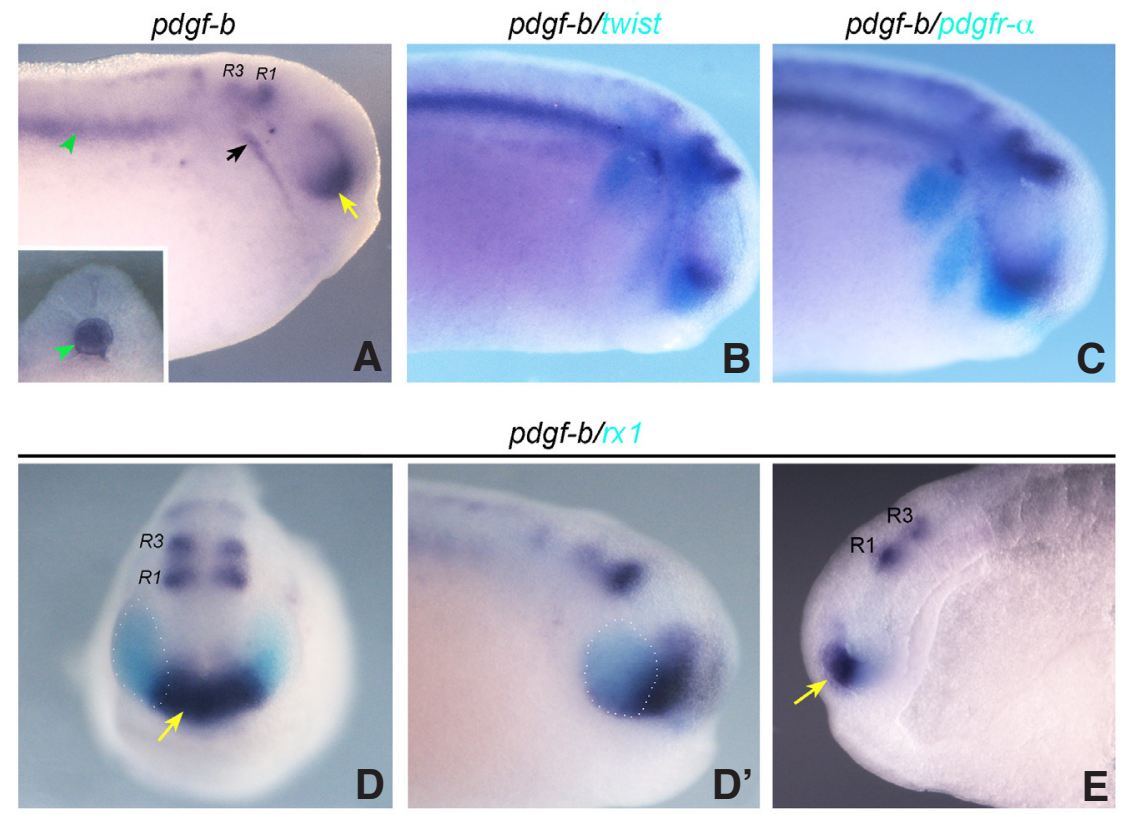

$p d g f-b / / \times 1$

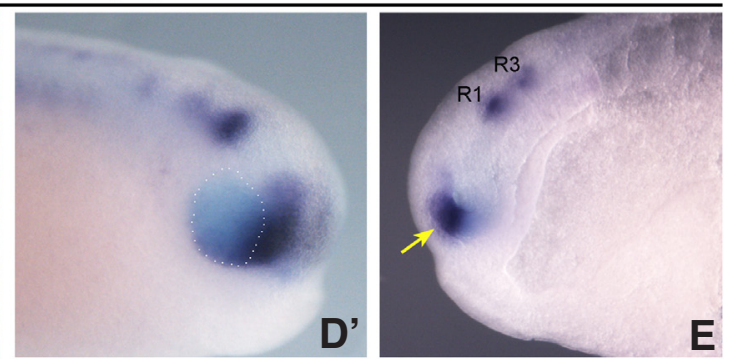


$r x 1$, observing that $p d g f-b$ partially overlapped $r x 1$ expression domain in the most medial domain of the eyefield (Fig. $3 \mathrm{H}$ ). These data established the presence of $p d g f-b$ mRNA within precise and confined areas of the developing CNS, suggesting a potential role for $p d g f-b$ in CNS development. This idea is supported also by previous data showing that the activity of the PDGF pathway is necessary to properly regulate oligodendrocyte progenitor cells (OPC) migration and proliferation (McKinnon et al., 2005) and that pdgf-boverexpression in vitro is sufficient to induce an OPC identity in neural progenitor cells (Appolloni et al., 2009). In addition, mutations in PDGF-B gene are responsible for causing primary familial brain calcification disease in humans (Keller et al., 2013).

Different studies demonstrated that PDGF signalling is implicated in Neural Crest Cells (NCC) development, but the mechanism of action remains controversial (Smith and Tallquist, 2010). In particular, the studies described so far were mainly focused on the role of pdgf-a and pdgfr- $\alpha$ during cranial NCC formation and migration (Eberhart et al., 2008, Ho et al., 1994), while a role for pdgf-b was supposed to be limited to a sub-population of cardiac NCC (Van den Akker et al., 2008). Cranial NCC are known to express pdgfr- $\alpha$ (Ho et al., 1994), that can activate an intracellular signalling cascade after the binding of pdgf-aa or pdgf-bb homodimers or the pdgf-ab heterodimer. In order to analyze the spatial relation between $p d g f-b$ expression domain and NCC, we performed double WISH using twist, a NCC marker (Fig. 3F), or pdgfr- $\alpha$ (Fig. 3G). These analyses showed that $p d g f-b$ mRNA was not expressed in NCC but in adjacent territories that can represent a source of pdgf-b during the early phases of NCC migration. In Xenopus, in fact, cranial NCC migrate from the mesencephalic and rhombencephalic regions of the CNS towards the branchial arches starting from late neurula (from stage 19). At the same stage, $p d g f-b m R N A$ reaches its maximum expression level (Fig. 2). At tailbud stages (Stage 24-25) pdgf-b mRNA was still present in the same CNS territories described before, in the notochord and in the facial placode (Fig. 4A and the inset). $P d g f-b$ expression domain remained in close proximity to NCC, that are still migrating at this stage, but never overlaps them as shown by the double WISH performed with twist or pdgfr- $\alpha$ (Fig. $4 \mathrm{~B}-\mathrm{C})$. The presence of $p d g f-b$ in the facial placode suggested that it might contribute to the correct formation of the facial nerve that is composed both by an ectodermal derivative, the placode, and NCC. In order to better define the most anterior expression domain of $p d g f-b$ in the CNS we repeated a double WISH at tailbud stage using the $r \times 1$ probe (Fig. 4 D-E). Pdgf- $b$ mRNA expression domain partially overlaps with the most anterior-medial $r x 1$ retinal expression domain (Fig. 4 D-D') and in a medial-sagittal view of an embryo at the same stage, a clear $p d g f-b$ labelling was visible also in the ventral telencephalon (Fig. 4E).

Taken together, these data unveiled the presence of $p d g f-b$ mRNA in neuroectoderm derived structures during embryogenesis. On the basis of pdgf- $b$ mRNA localization in a region adjacent to NCC expressing pdgfr- $\alpha$, it is tempting to speculate that $p d g f-b$ might also contribute to NCC development (Fig. 5). In this context it is interesting to remind that pdgf-b can bind to extracellular matrix components and that it can also stimulate hyaluronan synthesis, the most abundant glycosaminoglycan in the ECM surrounding cranial NCC. The developing NCC in Xenopus, in fact, produce hyaluronan and express the hyaluronan receptor CD44 (Casini et al., 2012). As the inhibition of the hyaluronan receptor CD44 could in turn inhibit pdgf-b induced cell growth (Li et al., 2007), it is tempting to hypothesize a possible role of pdgf-b in the activation of CD44 to instruct cranial NCC to keep on dividing during their migration. In the future, it will be interesting to investigate by functional experiments whether pdgf-b signalling could be required to control these two key phenomena in NCC development: cell division and migration.

\section{Conclusions}

The final result of this study was to unveil the gene expression pattern of $p d g f-b$ during embryonic development. The possibility to have a vertebrate model such as Xenopus, in which the pdgf- $b$ expression pattern is characterized, will allow future functional experiments aimed to clarify pdgf-b functions during brain and NCC development so far unsuspected. Moreover, overexpressing the PDGF-B mRNA in Xenopus embryos it will be possible to generate an alternative and useful model to study in vivo the pathogenic cellular mechanisms triggered by PDGF-B overexpression.

\section{Materials and Methods}

\section{Embryo preparation}

Animal procedures were performed in strict compliance with protocols approved by Italian Ministry of Public Health and of the local Ethical Committee of University of Pisa (authorization n.99/2012-A, 19.04.2012). Xenopus laevis embryos were obtained by hormone-induced laying and in vitro fertilization then reared in $0.1 \mathrm{X}$ Marc's Modified Ringer's Solution (MMR 1x: $0.1 \mathrm{M} \mathrm{NaCl}, 2 \mathrm{mM} \mathrm{KCl}, 1 \mathrm{mM} \mathrm{MgCl}$, 5 mM HEPES pH 7.5) till the desired stage according to Nieuwkoop and Faber (1967).

\section{Quantitative reverse transcription polymerase chain reaction (qRT- PCR)}

Total RNA was extracted from 10 wild type embryos for each developmental stage examined with Nucleospin $\circledast$ RNA (Macherey-Nagel) according to the manufacturer's instruction. cDNA was prepared by using iScript ${ }^{\mathrm{TM}}$ CDNA Synthesis Kit (Bio-Rad) and quantitative real-time PCR was performed using $\mathrm{iQ}^{\mathrm{TM}}$ SYBR ${ }^{\circledR}$ Green Supermix (Bio-Rad) according to the manufacturer's instruction. mRNA levels were normalized to the housekeeping gene ornithine decarboxylase (odc). Three different couple of primers were used: odc 
and pdgfr- $\alpha$ sequences were reported in (Bae et al., 2014), while primers for pdgf- $b$ were as follow: Forward 5'- GCTCTTCAATGCTCCTTTCC -3', Reverse 5'- TGTGCAATAAGAGGGCAGTG -3'.

\section{Whole mount in situ hybridization (WISH)}

Xenopus pdgf- $b$ sequence was obtained via RT-PCR using the following primers: forward, 5'- GAGATGTTCAAGAAGATCTCAG-3'; reverse, 5'- CACTACGTGACCAAAAGTTCTC-3'. PCR product sequence was aligned to pdgf-b sequence (Gene Bank: NM_001094466) and then cloned into pGEM-T easy vector (Promega). Digoxigenin (DIG) (Roche) labelled sense and antisense RNA probes were generated for $p d g f-b$ and Fluorescein (FLUO) (Roche) labelled antisense RNA probes were generated for twist, pdgfr- $\alpha$, engrailed2, krox20 and $r \times 1$. WISH was performed as described (Reisoli et al., 2010). We first revealed the DIG probe signal with BM Purple (Roche) and then the FLUO probe signal was visualized using BCIP substrate (Roche). After colour development, embryos were post-fixed and bleached under fluorescent light to remove the pigment.

\section{Acknowledgments}

We are grateful to J.P. Saint-Jeannet (pdgfr- $\alpha$ ), D. Alfandari (twist), $D$. Wilkinson (krox20), A. Hemmati Brivanlou (engrailed2) and M. Andreazzoli (rx1) for providing cDNAs for WISH. We thank M. Fabbri, G. De Matienzo and $E$. Landi for technical assistance and S. Di Maria for the frogs care. This work was supported by grants of "Ministero della Salute" (Italy) grant number GR-2008-1135643 and by Istituto Toscano Tumori Grant2010 "Use of in vivo model systems for the study of PDGF induced glioma progression".

\section{References}

ANDRAE, J., GOUVEIA, L., HE, L. and BETSHOLTZ, C. (2014). Characterization of platelet-derived growth factor-A expression in mouse tissues using a lacZ knock-in approach. PLoS One 9: e105477.

APPOLLONI, I., CALZOLARI, F., TUTUCCI, E., CAVIGLIA, S., TERRILE, M., CORTE, G. and MALATESTA, P. (2009). PDGF-B induces a homogeneous class of oligodendrogliomas from embryonic neural progenitors. Int J Cancer 124: 2251-2259.

BAE, C.J., PARK, B.Y., LEE, Y.H., TOBIAS, J.W., HONG, C.S. and SAINT-JEANNET, J.P. (2014). Identification of Pax3 and Zic1 targets in the developing neural crest. Dev Biol 386: 473-483.

BETSHOLTZ, C. (1995). Role of plateled-dervided factors in mouse development. Int. J. Dev. Biol. 39: 817-825

CALZOLARI, F. and MALATESTA, P. (2010). Recent insights into PDGF-induced gliomagenesis. Brain Pathol 20: 527-538.

CASINI, P., NARDI, I. and ORI, M. (2012). Hyaluronan is required for cranial neural crest cells migration and craniofacial development. Dev Dyn 241: 294-302.
DAMM, E.W. and WINKLBAUER, R. (2011). PDGF-Acontrols mesoderm cell orientation and radial intercalation during Xenopus gastrulation. Development 138: 565-575.

DEMOULIN, J.B. and ESSAGHIR, A. (2014). PDGF receptor signaling networks in normal and cancer cells. Cytokine Growth Factor Rev 25: 273-283.

EBERHART, J.K., HE, X., SWARTZ, M.E., YAN, Y.L., SONG, H., BOLING, T.C., KUNERTH, A.K., WALKER, M.B., KIMMEL, C.B. and POSTLETHWAIT, J.H. (2008). MicroRNA Mirn140 modulates Pdgf signaling during palatogenesis. Na Genet 40: 290-298.

HELDIN, C.H. (2013). Targeting the PDGF signaling pathway in tumor treatment Cell Commun Signal 11: 97

HELDIN, C.H. (2014). Targeting the PDGF signaling pathway in the treatment of non-malignant diseases. J Neuroimmune Pharmacol 9: 69-79.

HO, L., SYMES, K., YORDAN, C., GUDAS, L.J. and MERCOLA, M. (1994). Localization of PDGF A and PDGFR alpha mRNA in Xenopus embryos suggests signalling from neural ectoderm and pharyngeal endoderm to neural crest cells. Mech Dev 48: 165-74.

$\mathrm{HOCH}$, R.V. and SORIANO, P. (2003). Roles of PDGF in animal development. Development 130: 4769-4784.

KELLER, A., WESTENBERGER, A., SOBRIDO, M.J., GARCIA-MURIAS, M., DOMINGO, A., SEARS, R.L., LEMOS, R.R., ORDONEZ-UGALDE, A., NICOLAS, G., DA CUNHA, J.E. et al., (2013). Mutations in the gene encoding PDGF-B cause brain calcifications in humans and mice. Nat Genet 45: 1077-1082

LEVEEN, P., PEKNY, M., GEBRE-MEDHIN, S., SWOLIN, B., LARSSON, E. and BETSHOLTZ, C. (1994). Mice deficient for PDGF B show renal, cardiovascular, and hematological abnormalities. Genes Dev 8: 1875-1887.

LI, L., ASTERIOU, T., BERNERT, B., HELDIN, C.H. and HELDIN, P. (2007). Growth factor regulation of hyaluronan synthesis and degradation in human dermal fibroblasts: importance of hyaluronan for the mitogenic response of PDGF-BB. Biochem J 404: 327-336.

MCKINNON, R.D., WALDRON, S. and KIEL, M.E. (2005). PDGF alpha-receptor signal strength controls an RTK rheostat that integrates phosphoinositol 3'-kinase and phospholipase Cgamma pathways during oligodendrocyte maturation. $J$ Neurosci 25: 3499-3508.

NIEUWKOOP, P.D. and FABER, J. (1967). Normal Table of Xenopus laevis (Daudin) Elsevier: Amsterdam, The Netherlands.

REISOLI, E., DE LUCCHINI, S., NARDI, I. and ORI, M. (2010). Serotonin 2B receptor signaling is required for craniofacial morphogenesis and jaw joint formation in Xenopus. Development 137: 2927-2937.

SMITH, C.L. and TALLQUIST, M.D. (2010). PDGF function in diverse neural crest cell populations. Cell Adh Migr 4: 561-566

VAN DEN AKKER, N.M., WINKEL, L.C., NISANCIOGLU, M.H., MAAS, S., WISSE L.J., ARMULIK, A., POELMANN, R.E., LIE-VENEMA, H., BETSHOLTZ, C. and GITTENBERGER-DE GROOT, A.C. (2008). PDGF-B signaling is important for murine cardiac development: its role in developing atrioventricular valves, coronaries, and cardiac innervation. Dev Dyn 237: 494-503. 


\section{Further Related Reading, published previously in the Int. J. Dev. Biol.}

Unraveling new roles for serotonin receptor 2B in development: key findings from Xenopus

Michela Ori, Stefania De Lucchini, Giulia Marras and Irma Nardi

Int. J. Dev. Biol. (2013) 57: 707-714

http://dx.doi.org/10.1387/ijdb.130204mo

The role of angiogenic growth factors in organogenesis

Enrico Crivellato

Int. J. Dev. Biol. (2011) 55: 365-375

http://dx.doi.org/10.1387/ijdb.103214ec

Sequence and translation initiation properties of the Xenopus TGFbeta5, PDGF-A, and PDGF-alpha receptor 5 ' untranslated regions

A W van der Velden, A Los, $\mathrm{H} O$ Voorma and A A Thomas

Int. J. Dev. Biol. (2000) 44: 851-859

http://dx.doi.org/10.1387/ijdb. 11206326

Evidence that platelet derived growth factor (PDGF) action is required for mesoderm patterning in early amphibian (Xenopus laevis) embryogenesis J S Ghil and H M Chung

Int. J. Dev. Biol. (1999) 43: 329-334

http://dx.doi.org/10.1387/ijdb. 10470649

Role of platelet-derived growth factors in mouse development

C Betsholtz

Int. J. Dev. Biol. (1995) 39: 817-825

http://dx.doi.org/10.1387/ijdb. 8645566

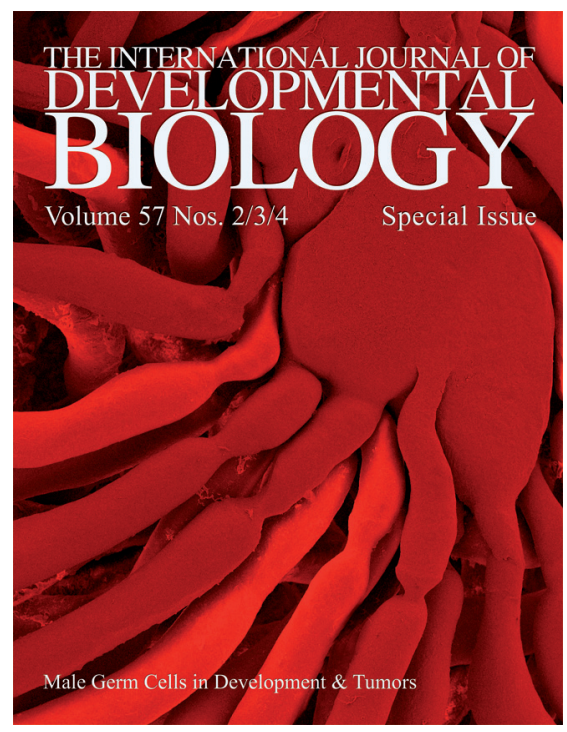

5 yr ISI Impact Factor $(2013)=\mathbf{2 . 8 7 9}$
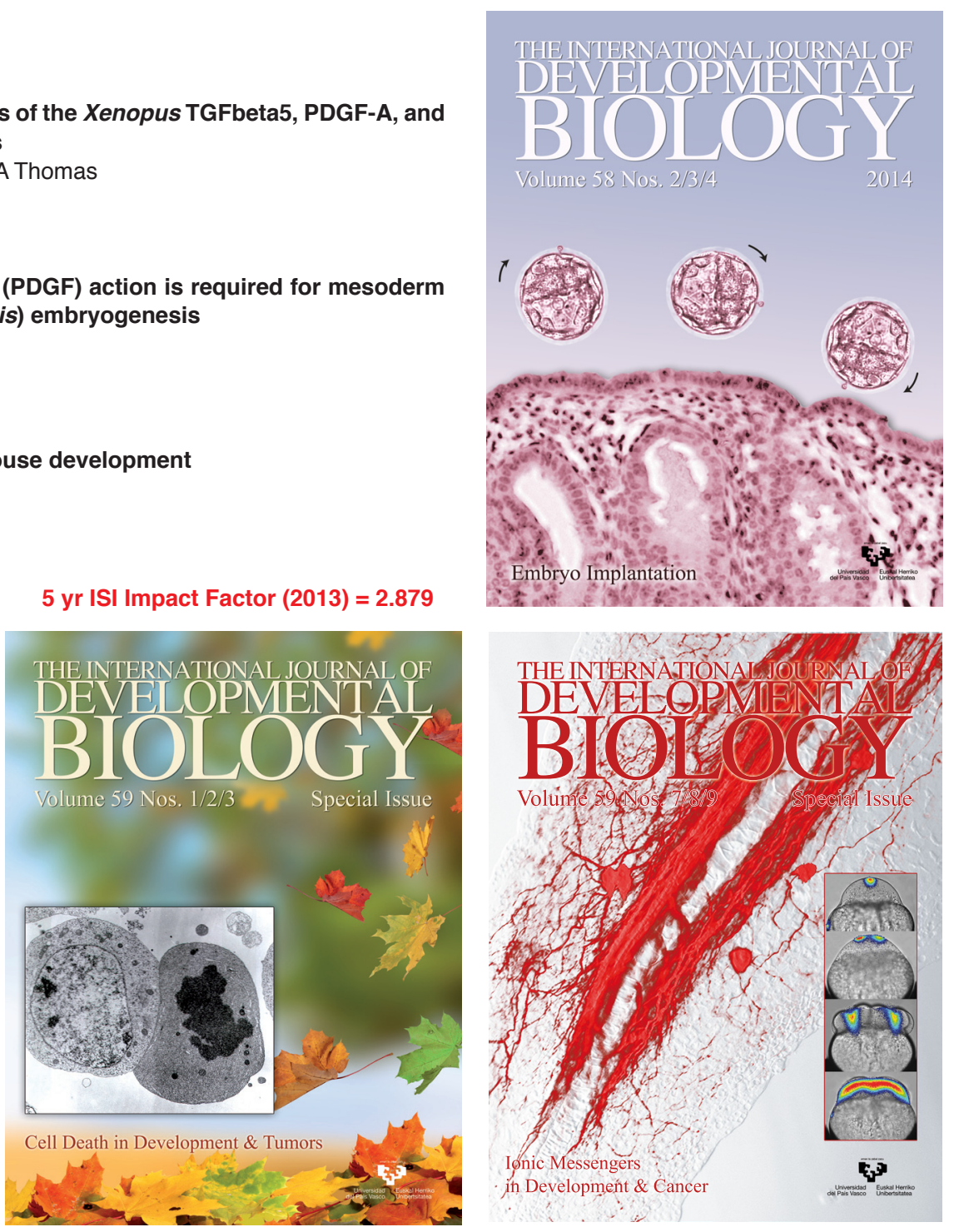